\title{
MATÍAS DE TORRES: PINTURAS EN PEÑARANDA DE BRACAMONTE Y HOYOS DEL ESPINO
}

\author{
Fernando Collar de CÁCERes \\ Universidad Autónoma de Madrid \\ fernando.collar@uam.es
}

\begin{abstract}
El pintor palentino Matías de Torres, activo en Madrid a caballo de los siglos XVII y XVIII, centró en buen grado su actividad profesional en encargos para templos y conventos de distintas localidades castellanas, ejemplo de lo cual son varias pinturas de temática mariana realizadas para Peñaranda de Bracamonte (Salamanca) y Hoyos del Espino (Ávila) que han que incorporarse, junto con una Inmaculada del convento de San Plácido y un dibujo de la Biblioteca Nacional, al catálogo de su obra.
\end{abstract}

Palabras clave: Matías de Torres; Pintura española; Dibujo; Siglo XVII; Peñaranda de Bracamonte; Hoyos del Espino.

\section{MATÍAS DE TORRES: PAINTINGS IN PEÑARANDA DE BRACAMONTE AND HOYOS DEL ESPINO}

The Palencian painter Matías de Torres, active in Madrid between the second half of the $17^{\text {th }}$ and early$18^{\text {th }}$ centuries, focused his professional activity on commissions from churches and convents in various Castilian villages, such as the several paintings of Marian themes for Peñaranda de Bracamonte (Salamanca) and Hoyos del Espino (Ávila), which now have to be incorporated into the catalogue of his work, as well as an Immaculate, in the convent of San Plácido, Madrid, and a drawing (Madrid, Biblioteca Nacional).

Key words: Matías de Torres; Spanish Painting; drawing; $17^{\text {th }}$ century; Peñaranda de Bracamonte; Hoyos del Espino.

Desde que hace cincuenta años Pérez Sánchez esbozara un primer estudio sobre el pintor Matías de Torres (Aguilar de Campoo, ca. 1631 - Madrid, 1711), con un apretado catálogo de su obra ${ }^{1}$, hemos asistido a un lento progreso en el conocimiento de la personalidad y de la creación pictórica de este artista palentino, cuya trayectoria profesional se desarrolló enteramente en Madrid. ${ }^{2}$

Claramente conectado con los maestros de su tiempo, aunque casi sin excepción al margen de los grandes encargos, don Matías de Torres, como le llama Palomino, emerge en el panorama de la

\footnotetext{
Pérez Sánchez 1965: 31-42.

2 Quesada /Jiménez 1996; Barrio Moya 1981a: 101; Barrio Moya 1981b: 456-458; Quesada 1994: 257-288; Barrio Moya 2008: 421-430; Nicolau Castro 2014: 88-92.
} 
pintura madrileña de la segunda mitad del siglo XVII con un estilo bien definido, deudor en cierto grado de las enseñanzas de Herrera el Mozo, a cuyo taller pasó desde el modestísimo obrador de Tomás Torrino, «pintor de tienda» ${ }^{3}$, en el que ingresó por razón de parentesco. Refiere en esto el tratadista cordobés cómo con el cambio y «con la asistencia a las célebres academias de aquel tiempo feliz y el trato de los pintores insignes que produjo aquella edad, mudó de estilo y entró en corrección de suerte que llegó a ser por su camino uno de los eminentes de esta facultad» ${ }^{4}$.

$\mathrm{Su}$ obra rompe en general no obstante con la manera dominante en la pintura postvelazqueña de los Carreño, Rizi, Antolínez, Solís o Camilo, de sentido retórico, estilo brillante y pincelada fogosa, heredera en lo técnico de lo veneciano y aun de la influencia flamenca, para avanzar por una vertiente en la que dominan los fuertes contrastes de luz, los contraluces de variado efecto, las medias sombras de apariencia acuarelada y fondos y formas de potente iluminación, jugando con todo ello en distintos planos, de desarrollo en general lateralizado, y definiendo con diverso matiz cuerpos y áreas de luminosidad o sombreado homogéneos hasta la planitud. Alcanza esto lo mismo a figuras siluetadas que a arquitecturas en semisombra o cegadas por la luz que llegan a adquirir así uniformidad tonal, si bien que no faltan propuestas como la inclusión de negras sombras y puntos de luminosidad intensa en un mismo cuerpo o composiciones de factura suelta y cromatismo múltiple, propias éstas, según se entiende, de sus primeros años. Es el caso de la Elevación de la Cruz (1668) de la Real Academia de San Fernando, inspirada tanto en Tintoretto como en Rubens, aunque faltan referencias para determinar si en el plano técnico sus creaciones tempranas fueron siempre así. En el extremo contrario, en cuanto al sentido de la luz y los valores tonales, están la Presentación de Jesús en el templo (1697) del Ermitage de San Petersburgo, el San José con el Niño $(1696)^{5}$ de las Descalzas Reales, la Adoración del Niño por san Jerónimo y santa Paula de la Academia de San Fernando o el San Lucas y el San Mateo de la Hispanic Society of America, que definen en esencia un momento de madurez, nitidez plástica y equilibrio compositivo.

Especialmente expresiva de su sentido de la pintura es desde luego la anécdota que recoge Palomino en relación con un cuadrito suyo de san Diego existente en el convento de la Victoria en el que predominaban de tal manera las áreas en sombra que sólo quedaba iluminado con nitidez el brazo de un mendigo, dado lo cual, estando de visita en el templo el pintor Francisco de Solís con cierto acompañante, como quiera que le preguntara éste sobre qué santo representaba el lienzo, respondió sarcástico Solís que se trataba de «San Brazo». Pero no todo es reductible a tan significativa particularidad. Se establecen en general dos momentos estilísticos bien diferenciados en su trayectoria, de los que el primero respondería a la conexión con el mundo pictórico de Herrera el Mozo, con valientes composiciones de dinamismo exacerbado, contrastes lumínicos, figuras en contraluz y fogoso colorido -a destacar en esto las extraordinarias pinturas de formato apaisado del retablo de Atienza (Historia de Esaú y Jacob, Jacob luchando con el ángel, la Construcción del Templo de Salomón, o Raquel escondiendo los ídolos)-, quedando el segundo, de tono más reposado y dibujo limpio, bajo la sugestión de Claudio Coello y Jiménez Donoso $^{6}$, con quienes tuvo ocasión de trabajar.

No faltan por lo demás en su obra otros referentes estilísticos más o menos ocasionales, de lo francés a lo flamenco o lo veneciano ${ }^{7}$, como va indicado, y en cuanto a lo español, de manera acaso indirecta y entre otros, Cano, de quien se muestra deudor en las figuras infantiles de algunas

\footnotetext{
${ }^{3}$ Entra en lo probable que el apellido «Torrino» no fuese sino in sobrenombre del tal pintor, que bien pudo llamarse Tomás de Torres si atendemos a su relación familiar.

${ }^{4}$ Palomino 1947: 1128.

${ }^{5}$ Sobre este cuadro, procedente de San Jerónimo el Real, puede verse en particular Kagané 2005: 171-174 y 503.

${ }^{6}$ Gutiérrez 1993: 130-133; Quesada 1994: 271.

7 Sobre estos aspectos Pérez Sánchez 1965: 34-35 y 37/ 1992: 331.
}

Arch. esp. arte, LXXXIX, 353, ENERO-MARZO 2016, 15-28 ISSN: 0004-0428, eISSN: 1988-8511, doi: 10.3989/aearte.2016.02 
de sus realizaciones más tardías (evangelistas de la Hispanic Society ${ }^{8}$, S. José con el Niño de las Descalzas Reales), como en la Presentación en el templo del Ermitage o en Cristo con la Samaritana de Atienza.

La producción artística de Matías de Torres fue en todo caso diversa, de las decoraciones efímeras en que participó por los años setenta a la pintura de batallas, países, cuadros de temática religiosa, retratos, pinturas murales -en colaboración en esto con Coello, Donoso y otros maestros madrileños-, trabajos de iluminación (privilegios, ejecutorias de hidalguía y similares) o pequeños cuadros con orlas florales y pinturejas, como refiere Palomino, quien pondera su facilidad en la invención y afirma que «tuvo habilidad en todo, aunque de muy corta fortuna» ${ }^{9}$.

Nos han llegado aunque no en gran número muestras que ilustran las más de estas realizaciones ${ }^{10}$, en algún caso tan sólo a través de diseños, como no de otra forma podía ser en lo que se refiere a sus labores efímeras, especialmente en esto en relación con la Entrada de María Luisa de Orleans (1679), y se ha podido ilustrar su labor como pintor de perspectivas, en pequeños caprichos históricos o bíblicos ${ }^{11}$, a contar entre las pinturejas que dice Palomino, quien da noticia de un trabajo de bien distinta naturaleza en ello como sería el retablo fingido de santa Lucía de la madrileña capilla del obispo, en San Andrés, de sentido efímero.

En el capítulo de las atribuciones resulta no obstante difícil situar en su trayectoria estilística y en relación sus criterios artísticos una obra como el Éxtasis de San Francisco de Padua (Valladolid. Museo Nacional de Escultura), distante en todo de sus planteamientos formales ${ }^{12}$ y sólo lejanamente emparentada como cuadro de altar con el lienzo de San Matías venerado por Carlos $V$ (Academia de San Fernando). Pero no faltan otras merecedoras de incorporarse sin reservas al catálogo de sus realizaciones.

Cabe mencionar entre éstas un pequeño lienzo de la Inmaculada Concepción $(110$ x $80 \mathrm{~cm})$ existente en el convento de San Plácido de Madrid y como tal registrado ${ }^{13}$, aunque nunca publicado, que es versión en mediano formato del perteneciente a los Padres Esculapios de España, en Madrid, de dimensiones muy superiores $(210$ x $148 \mathrm{~cm})$, y que cabe entender como su modelo ${ }^{14}$

${ }^{8}$ El carácter canesco del ángel de San Mateo determinó sin duda una primera atribución a Escalante. Véase Arnaiz 1991: 114

${ }^{9}$ Palomino 1947: 1129.

${ }^{10}$ Nada queda de sus pinturas murales y nada se sabe de sus ejecutorias de hidalguía y demás trabajos de iluminación, en los que se especializaría su hijo. Poco se conserva de otro lado de las numerosas pinturas que realizadas para san Jerónimo el Real (Adoración de San Matías por Carlos V, y Adoración del niño Jesús por San Jerónimo y santa Paula, Academia de san Fernando, Presentación en el Templo, Ermitage), y nada se ha podido identificar de lo pintado con certeza para el convento de la Victoria o de lo que pudo hacer para la Merced Calzada, aunque acaso recayó el encargo finalmente en Francisco de Solís, de acuerdo con la segunda opción así contemplada. Por lo que toca a la pintura de batallas, véase Quesada (1994. 277 y ss.), en atribución apoyada sobre fundamentos caligráficos. Hay copias de varias pinturas de la serie bíblica en el Museo provincial de Segovia, depositadas en parte en el Museo del obispado. En el Ermitage entraron ya en el siglo XIX varias pinturas de batallas suyas, registradas al menos en 1838 y cedidas en 1859 a Tsárkoye Seló (Kagané 2005: 96 y 176 y notas 17 y 222).

${ }_{11}$ Quesada 1994: 271-277. Se trata de pinturas de formato apaisado y poco más de una vara de ancho cuajadas de pequeñas figuras de notable viveza; así el Hallazgo del Moisés en el Niño y la Matanza de los Inocentes (Madrid, colección particular -como «pareja de caprichos arquitectónicos» en Madrid, Subastas Segre, 2006-12-19), la Entrada de Alejandro Magno en Babilonia (Madrid. Caylus) y la una Idolatría de Salomón (venta en Madrid. 2002-05-13).

${ }^{12}$ Véase VV.AA. 2002: 78-80. Su procedencia del convento vallisoletano de la Victoria se ha querido vincular a los trabajos realizados por el palentino para el madrileño de la misma orden de Mínimos, muy por encima de razones estilísticas.

${ }^{13}$ Fue requisado en la Guerra Civil por la CNT, el 29 de septiembre de 1937, depositado provisionalmente en el Museo del Prado y reintegrado en el convento con expediente de devolución SDPAN 141/5.

${ }^{14}$ Sobre esta pintura, Quesada, J. M. (J.M.Q.V.), «Inmaculada Concepción«, 2005: 256.

Arch. esp. arte, LXXXIX, 353, ENERO-MARZO 2016, 15-28

ISSN: 0004-0428, eISSN: 1988-8511, doi: 10.3989/aearte.2016.02 


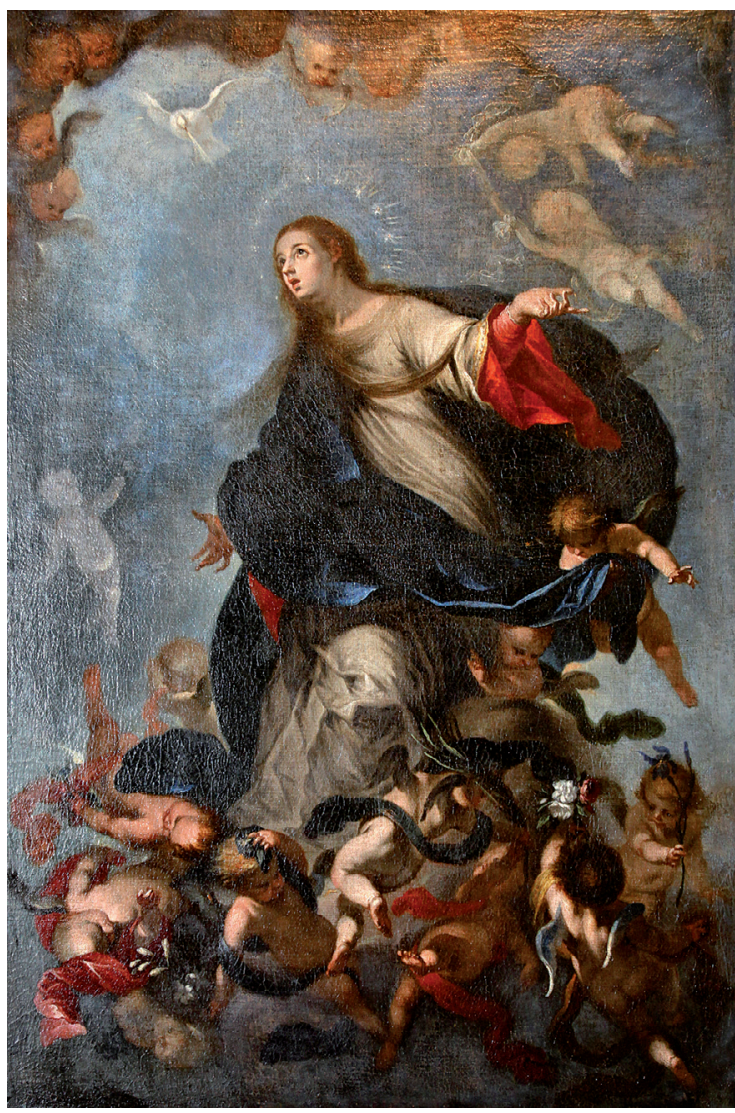

Fig. 1. Matías de Torres, Inmaculada Concepción. Benedictinas de San Plácido, Madrid. (fig. 1). Apenas existen entre ellos diferencias, la principal de las cuales está en la presencia de la paloma del Espíritu Santo, hacia la que dirige aquí la Virgen la mirada, dando más nítida razón a su expresión, rayana en el estupor, que unida a su disposición en giro lateral y su movimiento resulta sobre todo apropiada para su representación como Asunción. Desmiente que tal sea el tema el que varios de los angelitos que revolotean bajo ella lleven lirios, rosas o azucenas. Cambios mínimos hay en algunas de las ligeras telas flotantes con que juegan y en las sombras y brillos de algunos paños, pero sobre todo en el espacio que media entre la cabeza de las virgen y los querubines que presentes en lo alto, otorgándose con ello mayor amplitud a la composición por la parte superior. El aparato compositivo enlaza con los barrocos planteamientos Herrera el Mozo, por sus efectos en contraluz, oblicuidad figurativa y desaforado dinamismo, más allá de la propuesta aún bastante equilibrada en lo esencial de la Inmaculada de la Real Academia de San Fernando (cat. n. 70), acomodada en la figura mariana a modelos ya fijados.

\section{Historias marianas de Peñaranda de Bracamonte}

La llamada capilla de Loreto en el trasaltar de la iglesia conventual de las MM Carmelitas Descalzas de Peñaranda de Bracamonte (Salamanca) se adorna en los paños de sus lunetos -todos excepto los dos últimos carentes de vano- con una serie de lienzos pintados, tres de los cuales, de temática mariana, responden a un mismo criterio y hubieron de ser realizados ex profeso y a un tiempo para el lugar que ocupan, siendo los otros dispares y acomodados a sus paños de modo desigual, con mayor o menor molduraje ${ }^{15}$. Representan los tres primeros -sólo de éstos se tratará aquí- la Presentación de la Virgen en el templo, la Visitación - primer y segundo luneto del lado izquierdo, respectivamente- y el Nacimiento de la Virgen, primero del lado opuesto, que han sido ya estimados como de escuela madrileña, sugiriéndose a tal efecto el nombre de Diego de la Vega para los primeros y valorando como de otra mano y peor factura la Visitación ${ }^{16}$.

${ }^{15}$ En el segundo luneto del lado de la epístola, una mala copia de una pintura madrileña de la Inmaculada Concepción; en el tercero del lado del evangelio, lo que parece un tema teresiano, posiblemente Santa Teresa de Jesús asistida por los ángeles, con amplio molduraje para su adaptación al paño mural.

${ }^{16}$ «... en lo alto de los muros de la capilla, en un malísimo estado de conservación, colgados unos detrás de otros, una serie de lienzos entre los pudimos distinguir los temas siguientes: El Nacimiento, La Presentación en el Templo y la Visi-

Arch. esp. arte, LXXXIX, 353, ENERO-MARZO 2016, 15-28 ISSN: 0004-0428, eISSN: 1988-8511, doi: 10.3989/aearte.2016.02 


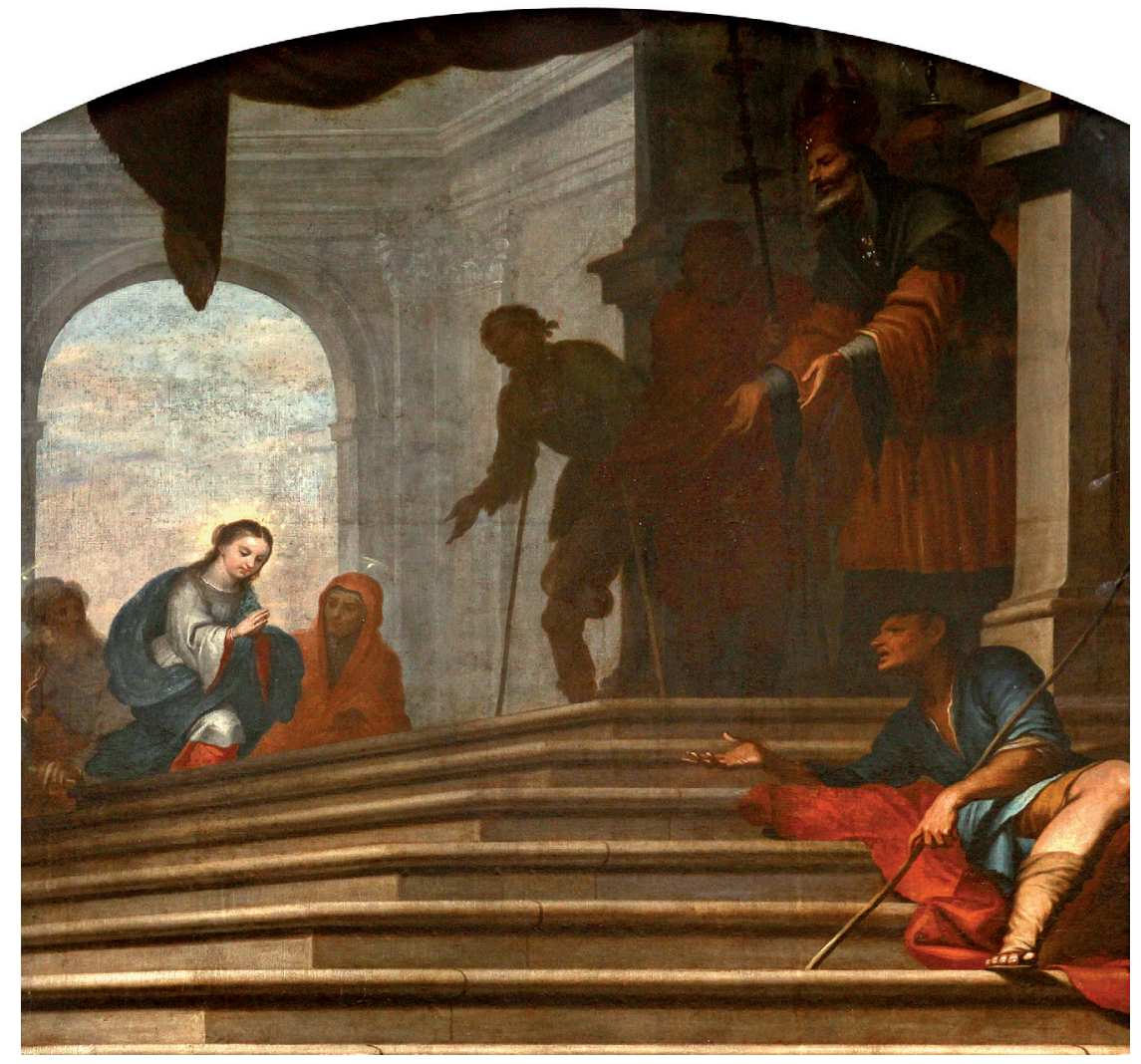

Fig. 2. Matías de Torres. Presentación de la Virgen en el templo. Peñaranda de Bracamonte (Salamanca), MM. Carmelitas Descalzas.

El estilo de estas tres pinturas conduce en realidad de manera indefectible a Matías de Torres, aunque ciertamente la Visitación parece alejarse de lo común de sus creaciones, debiendo tenerse en principio por obra de taller. Es de considerar en tal sentido el que conformen una serie incompleta, probablemente inconclusa, pues cabe entender que en principio se contemplaría la realización de seis cuadros, aunque no hay base documental que lo avale ni que explique las circunstancias o motivos de que se hicieran o existan sólo tres. No hay duda, de otro lado, que un encargo de esta naturaleza vendría precedido de un contacto previo en Madrid o su entorno con el Carmen Descalzo, a sumar así a las distintas órdenes (Mercedarios, Capuchinos, Jerónimos) para las que hasta la fecha se sabe trabajó.

La Presentación de la Virgen en el templo (fig. 2) es a todas luces del propio Torres, tanto en lo que respecta al criterio compositivo y los modelos figurativos como por el concepto lumínico y cromático y por factura. Al igual que las otras dos pinturas, su formato está definido en función del lugar que ocupa, con la particularidad en su caso de un encuadre compositivo en el que se ha tenido muy presente la circunstancia de su ubicación a una altura elevada. La escena está así vista desde abajo, quedando oculta la huella de los peldaños que sube la niña, lo que realza su presencia, as-

tación. Las dos primeras parecen obras madrileñas de calidad con fondos arquitectónicos como elementos fundamentales y quizá obra de Diego de la Vega. De peor calidad y sin duda de otra mano es la Visitación» (Casaseca Casaseca 1984: 2543-255). Nada consta en los estudios de E. Montaner ni en el Catálogo Monumental de Salamanca de Gómez Moreno.

Arch. esp. arte, LXXXIX, 353, ENERO-MARZO 2016, 15-28

ISSN: 0004-0428, eISSN: 1988-8511, doi: 10.3989/aearte.2016.02 


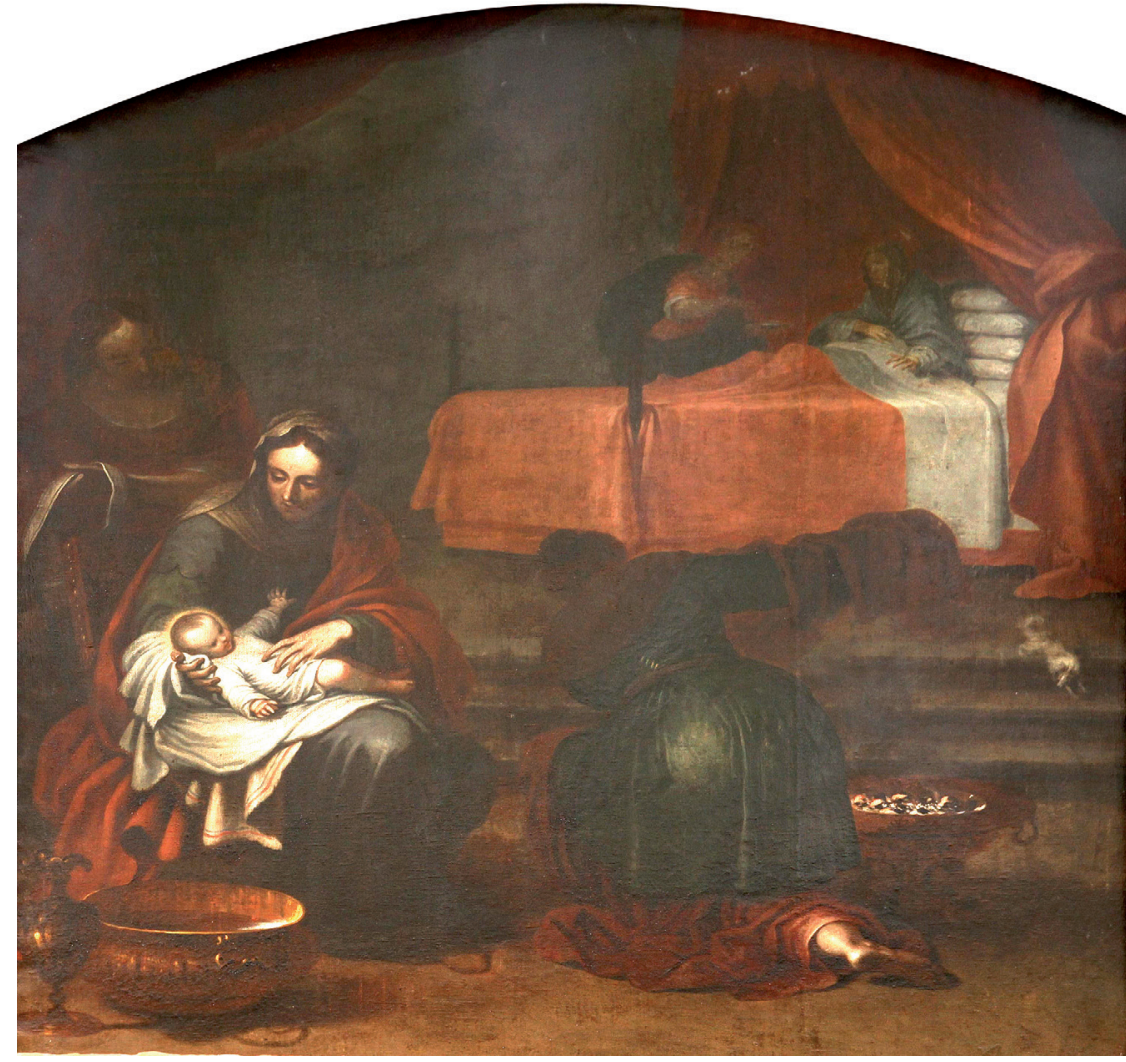

Fig. 3. Matías de Torres. Nacimiento de la Virgen. Peñaranda de Bracamonte (Salamanca), MM. Carmelitas Descalzas.

cendiendo casi de frente, y con ello la del sacerdote, que sale a su encuentro. La elección de una proyección oblicua confiere por otro lado a la composición una dimensión espacial que de otro modo se vería negada por su forzada contemplación, dando así profundidad a los escalones y desarrollando un marco arquitectónico descendente, de carácter clasicista, con pilastras corintias y un arco carpanel de acceso al recinto, cuya tenue definición contrasta con el oscuro paño que teatralmente cuelga en primer término y la mayor concreción plástica de los peldaños y de los elementos más próximos de la portada del templo. María, heredera directa de los modelos figurativos de Alonso del Arco, asciende con gesto humilde, con la mirada baja y las manos unidas, vestida con los colores de su condición inmaculada y coronada de doce estrellas, uniéndose con ello dos temas en uno. Sentado en las gradas destaca en primer plano el habitual mendigo, con el brazo extendido en demanda de caridad, muy en la línea con los personajes que en algunas de las pinturas del retablo de la Trinidad de Atienza aparecen como figuras adelantadas cuya acción y movimiento se orienta hacia el interior de la escena. Sólo él alcanza, por razón de proximidad, una definición plástica análoga a la de la Virgen. El sumo sacerdote, con las manos también adelantadas hacia la niña, queda parcialmente en sombra; sus acólitos, portando uno de ellos una pértiga, son poco más que una mancha rojiza de nítido contorno, y el tullido que aparece en pie al lado, apoyado en muletas y con la mano igualmente extendida, es casi sólo una expresiva silueta en contraluz, de inconfundible sello, mientras que san Joaquín y santa Ana, que acompañan a María hasta las gradas -visibles de medio cuerpo, en la medida que éstas, tras las que asoman, lo permiten-, responden a un abocetamiento plástico y cromático acorde con la habitual recurrencia a contraluces atemperados propia del artista. Pocas diferencias hay en todo ello con las figuras distantes del lienzo del Ermitage.

Arch. esp. arte, LXXXIX, 353, ENERO-MARZO 2016, 15-28 ISSN: 0004-0428, eISSN: 1988-8511, doi: 10.3989/aearte.2016.02 


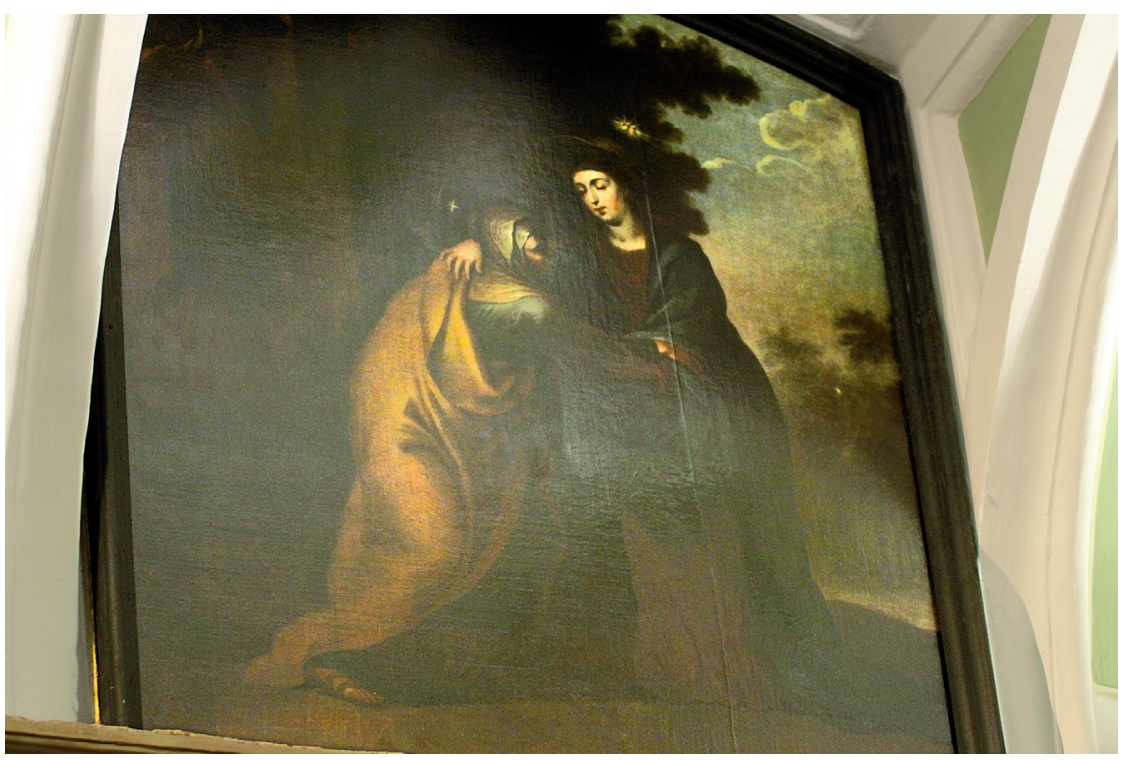

Fig. 4. Matías de Torres (taller). Visitación. Peñaranda de Bracamonte (Salamanca), MM. Carmelitas Descalzas.

El Nacimiento de la Virgen (fig. 3), en el luneto contrario, no participa de tal oblicuidad y encuadre, difiriendo también en lo tonal, pero adopta un planteamiento espacial dominado en primer plano por la figura de la nodriza con la niña, en el lado izquierdo, dando paso al espacio en penumbra de la alcoba de santa Ana, en el contrario, no muy lejos del sistema de planos en desarrollo lateral de algunas de sus más características creaciones. La luz llega de modo nítido sólo a la figura de la recién nacida, y de un modo más parcial a su nodriza, así como al pie que deja hacia atrás la sirvienta que vuelta de espaldas se gira para secar los pañales, verdadero «san brazo» de la escena -parafraseando a Solís-, con un punto complementario de luz propia en las ascuas del brasero. Delante de la rotunda figura de la nodriza descansan un gran barreño y una adornada jarra metálica, que hablan del primer baño de la recién nacida. Por detrás de la silla se asoma una segunda sirvienta, en figura abocetada, llevando en sus manos unos pañales secos. El fondo de la estancia, de oscuridad algo menor, es ocupado por el lecho de santa Ana, de dosel y ropas rojizas, en un plano ligeramente más elevado cuyos tres escalones son salvados por un juguetón perrillo, en claro guiño a la cotidianidad, como de otro modo el que ladra nervioso en la temprana Elevación de la Cruz de la Academia de San Fernando. Varios elementos suscitan por lo demás una inspiración compositiva en algunas de las más difundidas estampas del tema, pero no hay dependencia directa de ninguna de las conocidas, volcando sin duda Torres su sello personal.

Menos característica de su estilo resulta la Visitación (fig. 4), de una oscuridad similar, aunque la escena tiene lugar en un exterior, a las puertas de la vivienda de santa Isabel, y en la que los brillos, las sombras y la anciana figura de la santa tienen que ver sin duda con el cuadro anterior y con la Hemorroísa de Atienza, y las redondas facciones de María, tocada con sombrero peregrino, remiten a la Anunciación del mismo retablo alcarreño, aunque no puede negarse que es su pertenencia a esta serie mariana lo que mueve en esencia a pensar en el pintor de Aguilar de Campoo o, ya en mayor medida, en su taller. Como específico de su estilo, las plumosas copas de los árboles, el celaje, el sombreado de las ropas de la santa prima de María y en algún grado el sentido del color. Es obra, de cualquier modo, oscurecida y muy afectada en sus barnices, lo que dificulta su exacta valoración.

Arch. esp. arte, LXXXIX, 353, ENERO-MARZO 2016, 15-28

ISSN: 0004-0428, eISSN: 1988-8511, doi: 10.3989/aearte.2016.02 


\section{Pinturas del retablo de Hoyos del Espino}

El recientemente restaurado retablo mayor del la iglesia parroquial de Nuestra Señora de la Asunción en la localidad abulense de Hoyos del Espino, al pie de la Sierra de Gredos, aloja en las calles laterales de su segundo cuerpo un par de lienzos de temática mariana, conforme a su advocación, que se deben a ciencia cierta al pincel de don Matías de Torres y no tomados hasta el momento como tales en consideración. La notoria conexión que ambas composiciones con modelos ajenos, en muy superior grado a lo que por lo común ocurre en la obra del palentino, sugiere que, si bien ser trata de pinturas con sentido del dibujo propio de un momento avanzado de su trayectoria, probablemente anteceden a las de Peñaranda de Bracamonte y a las realizaciones de los años noventa. Así se entiende por la cronología de la obra.

En lo documental, lo único que reflejan las cuentas parroquiales es que el retablo, de traza clasicista, fue realizado a partir de 1649 y asentado en $1653^{17}$, tasándolo entonces el ensamblador Pedro Ribero ${ }^{18}$, y bajándose en 1672 para su dorado, labor ésta que estuvo al cuidado del dorador Andrés de Medina ${ }^{19}$ (fig. 5). En ningún momento se hace referencia a la parte pictórica, pero en buena ley los dos lienzos serían dispuestos a lo sumo ya aquí por estas fechas.

La Purificación de la Virgen (fig. 6), en el lado del evangelio, es de ambas la pintura que de manera más patente exhibe rasgos y modelos propios de Matías de Torres. La composición se ordena en una disposición enteramente lateral, con Simeón sobre las gradas del templo, inclinado hacia María y teniendo ya al Niño en sus manos, y la Virgen arrodillada en los últimos escalones, aún con los brazos extendidos, tras la entrega, teniendo el cestillo con la pareja de tórtolas ofrendadas ante sí. Es escena que se ajusta así a cánones bien establecidos, sin entrar en el momento decisivo de la entonación del evangélico Nunc dimittis por parte de Simeón, de escasa implantación en España, que adoptaría precisamente Torres en la muy distinta versión del Ermitage, en la que retoma no obstante diversos elementos de ésta. Tras la Virgen figura en un segundo plano José y, acompañando al sacerdote, tres acólitos o servidores del templo, que se asoman curiosos junto a las columnas, distinguiéndose más allá de las escaleras, en un plano más distante, un hombre y una mujer de edad, identificable con la profetisa Ana (Lc. 2, 36-40).

Es pintura de equilibrado sentido compositivo y notable factura en la que poco cabe decir en su caso de la creatividad de su autor, ya que en esencia se trata de una versión simplificada de la Purificación de la Virgen pintada en 1640 por Simon Vouet, heredera de la de Palma el joven ${ }^{20}$, que probablemente conoció a través de una copia o estampa, aunque no deja de haber elementos en común con la más compleja Presentación de la Virgen en el templo de Peñaranda de Bracamonte (arco, cortinaje, diseño de las gradas, sombras, encuadre ascendente, etc.) y no cabe desestimar el referente de las pinturas de Solís para el convento madrileño de los Trinitarios Descalzos. Las di-

17 A.D.Av. Hoyos del Espino. «Libro de quentas de la Yglesia de Hoyos del espino enpieça en 1653«, cuentas de 1653 y 1654. Incluye unas primeras cuentas de 1649 a 1652, quedando constancia en ellas de que ya entonces se pagó por la madera cortada para el retablo. Éste venía a sustituir al retablo de pintura mencionado en distintos inventarios $\left(1^{\circ}\right.$ y $3^{\circ}$ ), que en la parte de talla fue realizado en 1541 por Hernández Buenadicha.

${ }^{18}$ Las cuentas de 1654-55 registran en este capítulo pagos por obras en la capilla mayor y lo gastado en cal, telas, madera y clavazón. Las de 1670 -1671 detallan lo referido a la caja de la imagen de la titular, dispuesta en el retablo; su costo 6.936 mrs. Ribero dirigió por 1605 las obras de la torre de la iglesia de la extremeña localidad de Hoyos, en la sierra de Gata, a instancias del maestro mayor de la catedral de Coria, Diego González (García-Mogollón 2006: 38).

${ }^{19}$ El mismo libro de fábrica detalla en lo tocante a 1674-75: «retablo. Mas trescienttos y ochentta y nueue Rs que el dho $\mathrm{My}^{\mathrm{mo}}$ pago a quenta del rretablo a andres de Medina. dorador q doro el rretablo solo fue por el dorador $\operatorname{tr}^{\mathrm{a}}$ Rs lo demas por asentarle». Sigue otro pago de 119.280 maravedís al mismo maestro a cuenta de los 1.800 reales en que se concertó la obra y 91 reales pagados a dos oficiales que le asistieron.

${ }^{20}$ Mayer, A. L. (1947: 471) percibió, de igual modo, una posible influencia de la pintura academicista francesa en la muy distinta versión de Ermitage; citado por Pérez Sánchez. (1965: 34-37).

Arch. esp. arte, LXXXIX, 353, ENERO-MARZO 2016, 15-28 ISSN: 0004-0428, eISSN: 1988-8511, doi: 10.3989/aearte.2016.02 


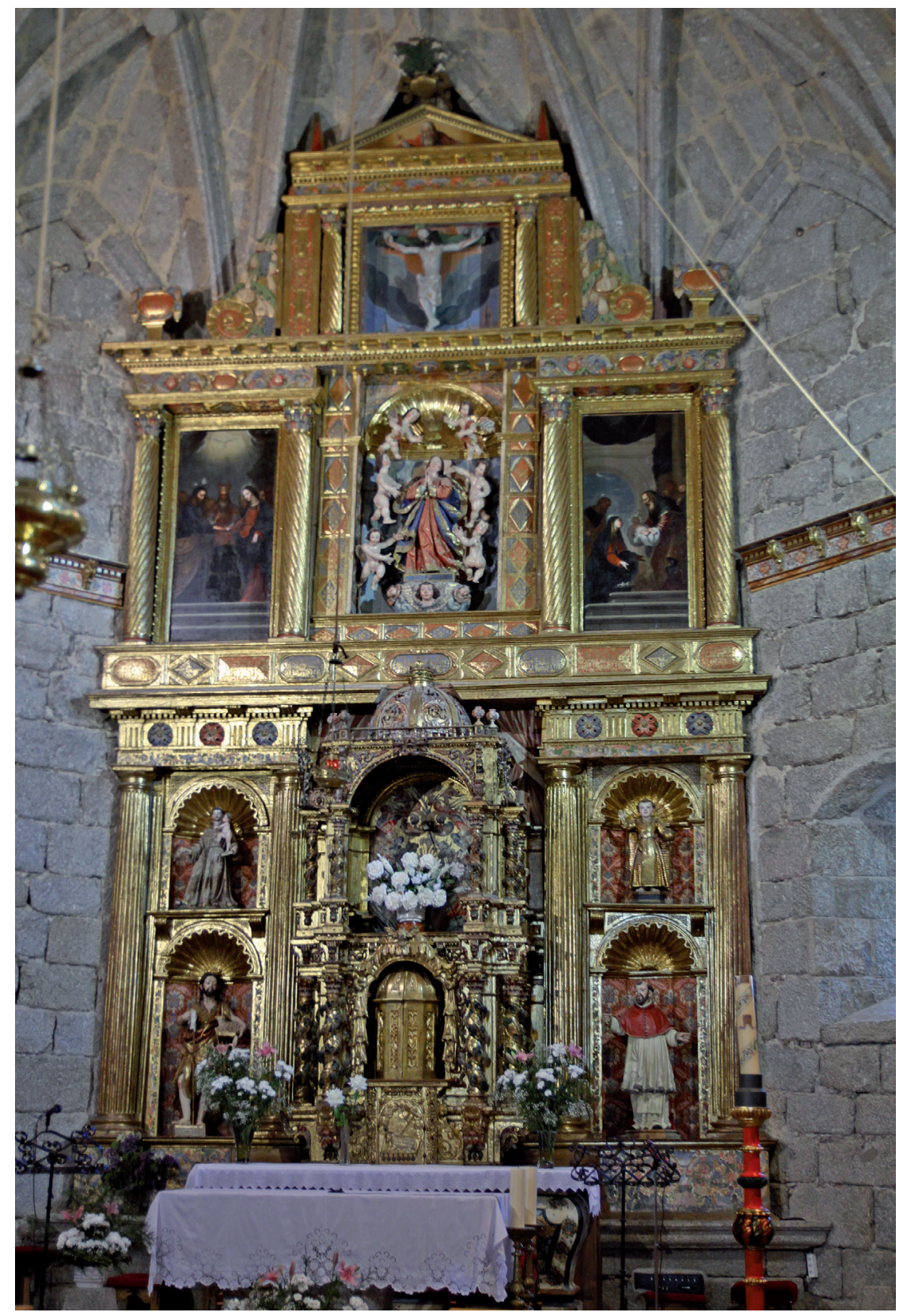

Fig. 5. Hoyos del Espino (Ávila), Retablo mayor. (1649-1653), iglesia de la Asunción.

ferencias formales y cromáticas respecto del lienzo de Vouet son desde luego importantes, con una radical reducción en el número de los presentes y una absoluta simplificación del marco arquitectónico, de limpias formas clásicas, como en Solís; pero las deudas figurativas van más allá de María, Simeón o el Niño y alcanzan incluso a los personajes que asoman tras de las gradas. El resultado final es sustancialmente otro, siquiera sea por la saturación cromática y el sentido del claroscuro, con una característica definición en sombra el perfil facial de María, propia de Cavallino.

Arch. esp. arte, LXXXIX, 353, ENERO-MARZO 2016, 15-28

ISSN: 0004-0428, eISSN: 1988-8511, doi: 10.3989/aearte.2016.02 


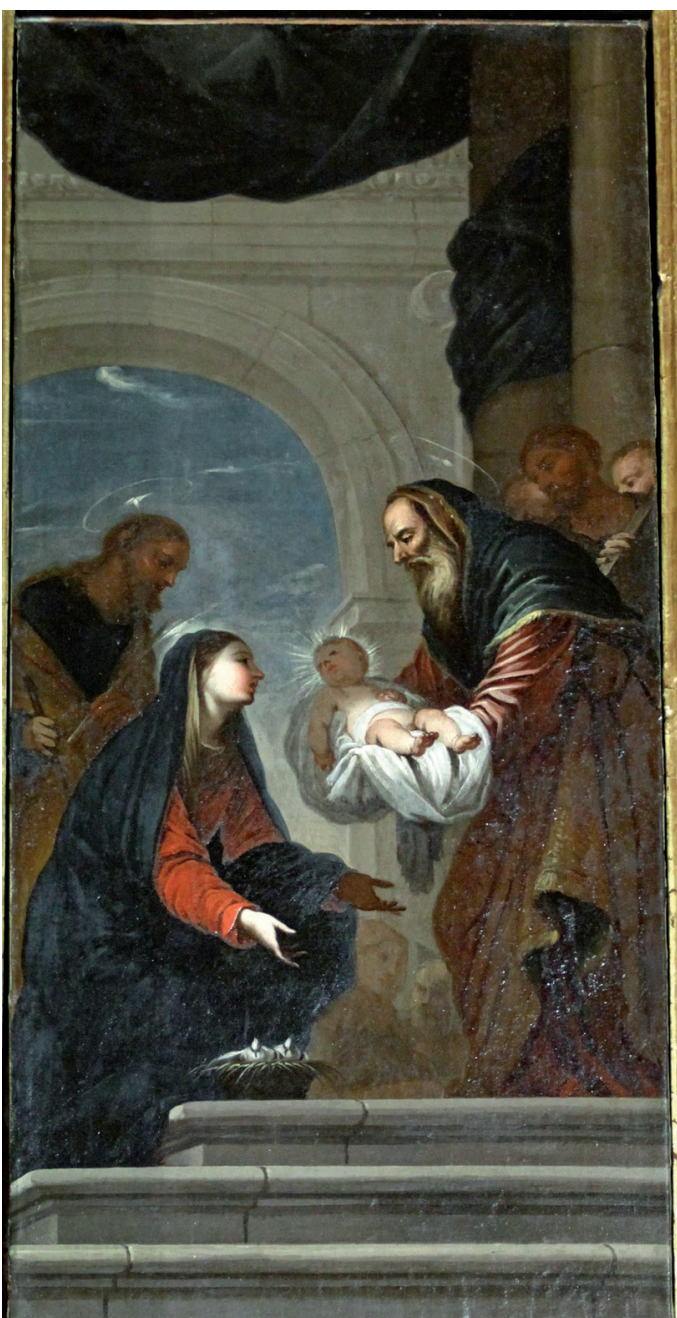

Fig. 6. Matías de Torres. Presentación de la Virgen en el templo. Hoyos del Espino (Ávila), iglesia de la Asunción.

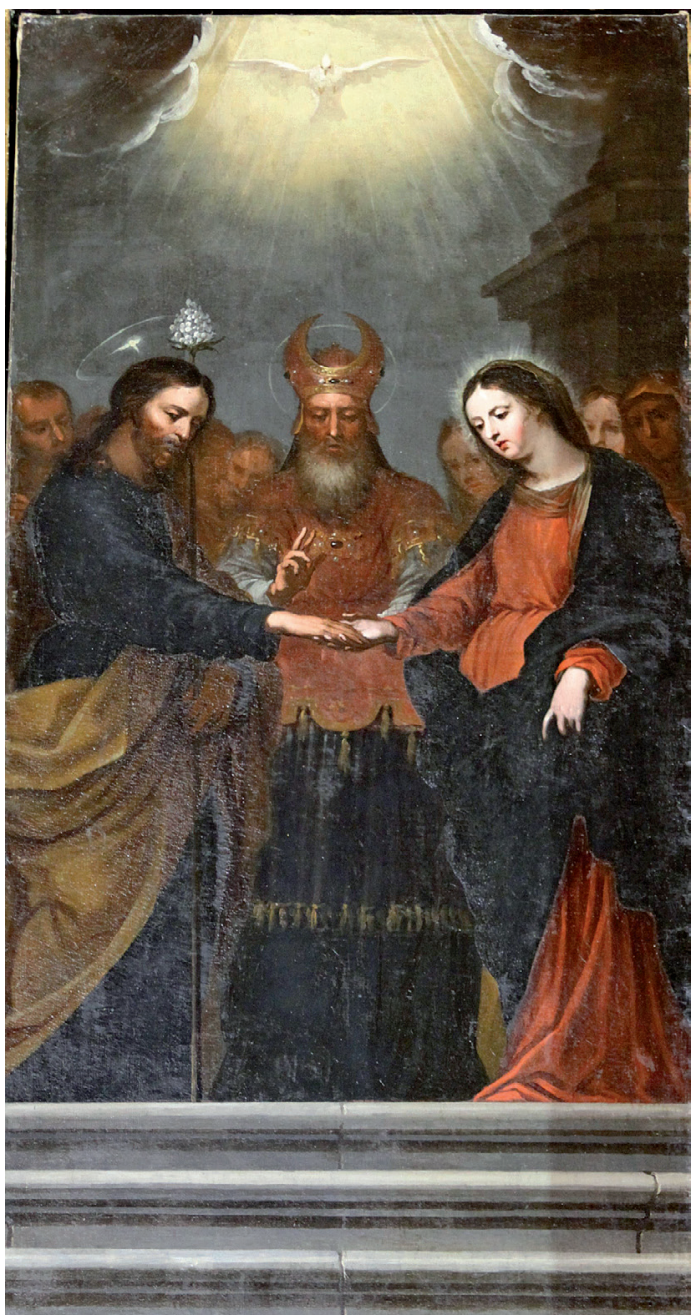

Fig. 7. Matías de Torres. Desposorios de la Virgen. Hoyos del Espino (Ávila), iglesia de la Asunción.

En ello, como en algunos elementos figurativos y el sentido del color, hay una estrecha relación con la Purificación de la Virgen del Ermitage, de muy distinta naturaleza compositiva ${ }^{21}$. En lo iconográfico es de reseñar la singular representación nimbada Simeón, en figura de particular nobleza y expresividad. En cuanto al Niño, cabe advertir que en buen grado anticipa el de la Adoración del Niño por san Jerónimo y santa Paula (Academia de S. Fernando) o el San José con el Niño de las Descalzas Reales (Madrid).

${ }^{21}$ El particular recurso del sombreado de un perfil facial está asimismo en la estilizada figura del caballero de presencia destacada en el dibujo de María Estuardo conducida al patíbulo (M. del Prado).

Arch. esp. arte, LXXXIX, 353, ENERO-MARZO 2016, 15-28 ISSN: 0004-0428, eISSN: 1988-8511, doi: 10.3989/aearte.2016.02 
Los Desposorios de la Virgen (fig. 7), segunda de las pinturas del retablo, responde a la más convencional idea compositiva del tema, acorde con los viejos modelos de Durero e Israel van Meckenen, aunque casi desprovista de todo enmarcamiento arquitectónico. La escena adolece así de total frontalidad compositiva y de excesivo estatismo, quedando reducida al solo grupo de la Virgen y José, con el sumo sacerdote en el centro, y cinco o seis cabezas de testigos que agrupados por sexos se asoman desde un segundo plano, con limitado efecto de profundidad. Como referente espacial, tres escalones, siempre de un mismo diseño, en visión estrictamente frontal, definiendo el plano en cuyo borde en el que se asientan las figuras, con el basamento y arranque de una gran columna. En lo alto, un destello de luz, y el Espíritu Santo, flotando sobre la cabeza de los congregados. Más allá del sentido convencional de la composición, de genéricas similitudes con no pocas versiones del tema o del lejano patrón de las estampas germánicas, todo indica que el pintor se sirvió aquí de un modelo específico a tenor de las estrechas semejanzas que se detectan con alguna modesta versión quinientista ${ }^{22}$.

\section{Dibujo de un apóstol}

Uno de los aspectos en más estudiados en los últimos años en relación con Matías de Torres es el que toca al dibujo ${ }^{23}$, capítulo en el que cabe agrupar de un lado creaciones de neta y equilibrada definición lineal, en tinta y aguada sepia, con figuras por lo común muy estilizadas, como en buena parte de los diseños para las decoraciones de la Puerta del Sol con motivo de la entrada de la reina, y de otro aquéllas de trazo más nervioso y figuras agitadas, en igual técnica, como la serie de reyes y caudillos célebres para un juego de naipes o un par de escenas mitológicas inspiradas en Tempesta (British Museum), aunque también algunos dibujos de criterio más próximos a sus realizaciones pictóricas, jugando con fuertes sombras entintadas y áreas luminosas, como ocurre en el de Rebeca y Eliezer (Albertina de Viena).

Nada que ver con todo ello ofrece sin embargo un dibujo de Santo Tomás, apóstol de la Biblioteca Nacional de Madrid (dib. 3963; lápiz grafito, en papel verjurado 176 x $141 \mathrm{~mm})^{24}$, catalogado como obra española de los siglos XVIII-XIX, que estimamos suyo (fig. 8). Representa al santo apóstol de medio cuerpo y de avanzada edad, barbado y calvo, con amplio manto y gesto parlante, que gira su rostro hacia la derecha, en sentido opuesto al cuerpo, y señala expresivamente con la mano izquierda hacia el lado contrario, sosteniendo con la otra la lanza, en referencia a su martirio.

Realizado con lápiz negro sobre papel verjurado, es obra de una factura suave y suelta, en la que se definen las formas con trazos sutiles y un sombreado tenue que oscurece también en parte del fondo y que envuelve a la figura en una atmósfera poco nítida. Se separa así de casi cuanto se conoce o mejor se conoce de su obra dibujística. Dista sin embargo de ser una absoluta excepción, pues guarda estrechísima relación con el dibujo de San Felipe (Granada, colección particular), recientemente catalogado como suyo ${ }^{25}$ y con el que concuerda en todas sus particularidades técnicas

${ }^{22}$ Véase la pequeña tabla del tema en una de las calles laterales del retablo de la Comunión de la Virgen del monasterio toledano de San Pablo (MM. Jerónimas), del entorno de Hernando de Ávila, atribuida sin gran fundamento a Diego de Aguilar. Los términos de la composición y gestos de las figuras quedan invertidos en la versión de Alonso Cano (Museo Goya, Castres).

${ }^{23}$ Entre otros, Brown 1975: 235 y ss., rectificado por Hoffmann-Samland 2014: 140 y 250; Pérez Sánchez 1986: 274-275; Zapata 2000, passim; Muller 2006: 202-204; Zapata Fernández de la Hoz 2010: 482-497; McDonald 2012: 112; McDonald 2013: 88-91.

${ }^{24}$ En el reverso, en tinta: «Madrid, 1879».

${ }^{25}$ Gutiérrez Pastor, 2008: 162.

Arch. esp. arte, LXXXIX, 353, ENERO-MARZO 2016, 15-28

ISSN: 0004-0428, eISSN: 1988-8511, doi: 10.3989/aearte.2016.02 


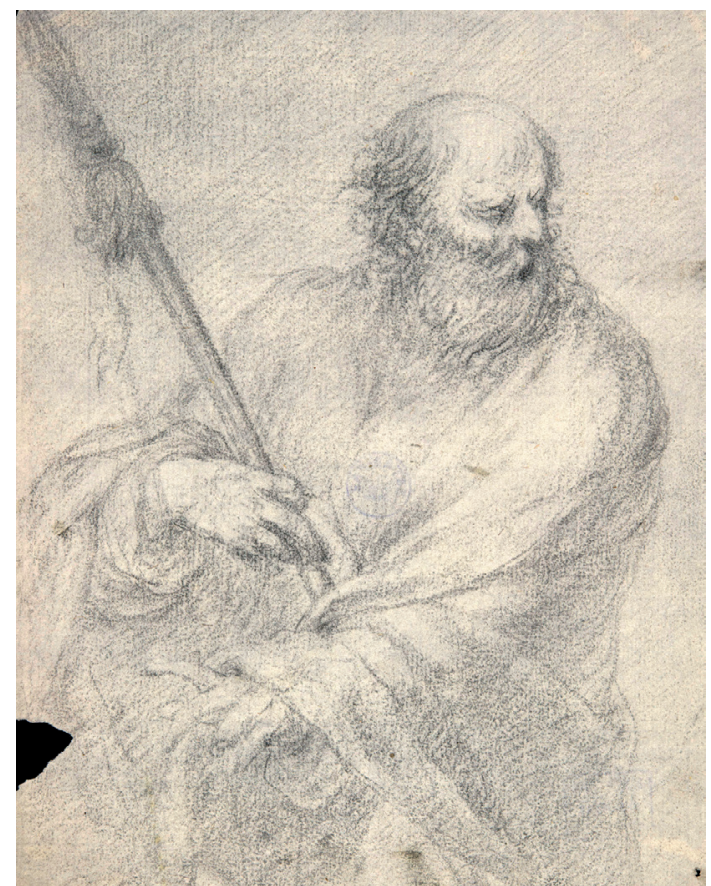

Fig. 8. Santo Tomás, dibujo. Madrid, Biblioteca Nacional.

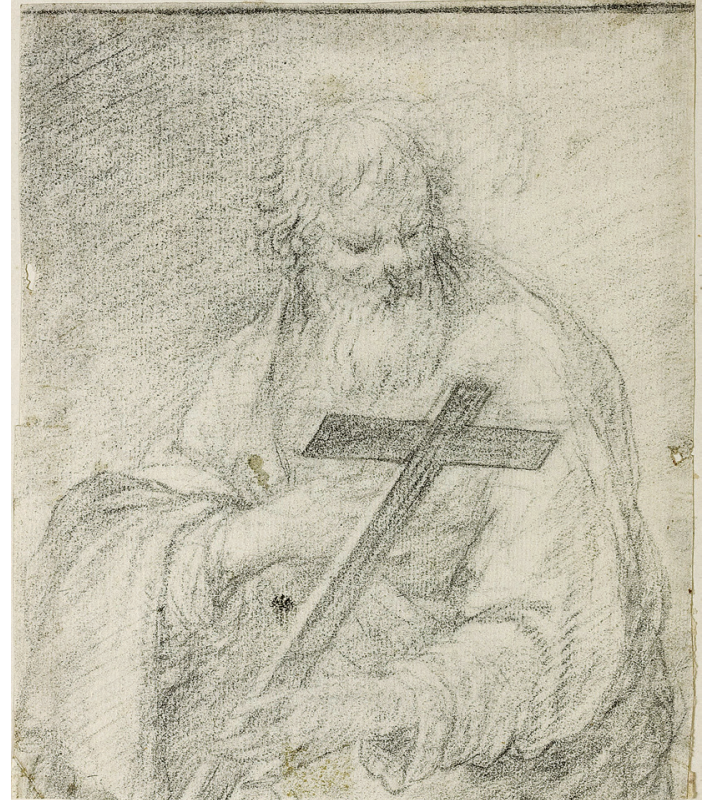

Fig. 9. San Felipe. Granada, colección particular.

y formales, de sus medidas y calidad del papel $^{26}$ a la concepción figurativa y la factura (fig. 9), de lo que hay que concluir que formaron a no dudarlo parte de una misma serie. Se entiende así que Matías de Torres los concibió como preparación de un apostolado, que bien pudiera ser el que menciona Palomino ${ }^{27}$ en el coro de San Jerónimo el Real de Madrid que aún alcanzó a ver Ponz, quien lo describe como «lo principal« de mano del pintor allí existente ${ }^{28}$. La veracidad y viveza figurativa, patente de modo particular en la expresión parlante de santo Tomás, cuadra a la perfección con el juicio emitido por Ponz sobre las pinturas. En lo fisonómico, con su larga barba de aspecto nebuloso, es cabeza que se aproxima de modo notorio a la del adorante san Jerónimo del lienzo de la Academia de San Fernando que fue del mismo monasterio madrileño, lo que de algún modo viene en apoyo de la idea de que los dibujos fueron parte del trabajo preparatorio de las pinturas realizadas por el artista ya en su madurez para el citado monasterio madrileño. Figurativamente no hay duda de que el modelo de referencia hubo de ser una pintura de Herrera el viejo cuyo dibujo preparatorio es el supuesto San Tadeo del apostolado de la Kunsthalle de Hamburgo, de muy distinta factura ${ }^{29}$, que pudo conocer Matías de Torres en su relación con Herrera el Mozo.

\footnotetext{
${ }^{26}$ La inexistencia de marca de agua, como la sólo parcialmente visible en el dibujo de San Felipe, se debe a tratarse de hojas recortadas.

${ }^{27}$ Palomino 1947; 1129.

${ }^{28}$ Ponz, V.22 (1988:33). Literalmente: «Lo principal de Matías de Torres es un apostolado en el coro«.

${ }^{29}$ Sobre la plasmación en lienzo de algunos de los dibujos de la serie en el apostolado incompleto del museo de Córdoba, Pérez Sánchez/Navarrete Prieto, 1996: 365-387.
}

Arch. esp. arte, LXXXIX, 353, ENERO-MARZO 2016, 15-28 ISSN: 0004-0428, eISSN: 1988-8511, doi: 10.3989/aearte.2016.02 


\section{BIBLIOGRAFÍA}

Arnaiz, José Manuel (1991): «Cuadros inéditos del siglo XVII español». En: Anuario del Departamento de Historia y Teoría del Arte (UAM), III, pp. 109-124.

Barrio Moya, José Luis (2008): «Nuevas aportaciones a la biografía del pintor palentino Matías de Torres (1635-1711)». En: Publicaciones de la Institución Tello Téllez de Meneses, n. 79, pp. 421-430.

Barrio Moya, José Luis (1981a): «Las deudas del pintor Matías de Torres». En: Archivo Español de Arte, LIV, n. 213, p. 10.

Barrio Moya, José Luis (1981b): «Noticia sobre Alonso Cano. Andrés de Vargas, Matías de Torres y Pompeyo Leoni en el convento de Atocha». En: Archivo Español de Arte, LIV, n. 216, pp. 456-458.

Brown, Jonathan (1975): «Drawings by Herrera the Younger and a follower». En: Master Drawings, 13.3, pp. 235-240.

Casaseca Casaseca, Antonio (1984): Catálogo monumental del Partido Judicial de Peñaranda de Bracamonte (Salamanca). Salamanca, Madrid, Ministerio de Cultura.

García-Mogollón, Florencio Javier (2006): «Hoyos. Arquitectura popular y arte religioso en una localidad del norte de Extremadura». En: Cuariensia, n. 1, pp. 6-66.

Gómez Moreno, Manuel (1965): Catálogo monumental de Salamanca. Madrid, 2 vols.

Gómez Moreno, Manuel (1983): Catálogo monumental de Ávila (1900-1901) Madrid, Ávila, Fundación Gran Duque de Alba, Ed. de Aurea de la Morena y Teresa Pérez Higuera; 3 vols.

Gutiérrez, Ismael (1993): «Matías de Torres. San Mateo; San Lucas». En: De la Edad Media al Romanticismo. Madrid, Caylus Anticuario, S.A., pp. 130-133.

Gutiérrez Pastor, Ismael (2008): «Matías de Torres (1635-1711). San Felipe». En: A. Ansón Navarro y Ricardo Centellas Salamero (coms.): El dibujo español del Renacimiento a Goya. La colección de la Reina María Cristína de Borbón. Catálogo de la exposición. Zaragoza. Diputación de Zaragoza, pp. 162163.

Hoffmann-Samland, Jens (com.) (2014): The Spanish Gesture. Drawinf from Murillo to Goya in the Hambueger Kunsthalle Meadows Museum. Dallas.

Kagané, Ludmila (2005): La pintura española del museo del Ermitage. Siglos XV a comienzos del XIX. Sevilla, Museo del Ermitage y Fundación El Monte.

Mayer, August L. (1947): Historia de la pintura Española. Madrid, Espasa Calpe.

McDonald, Marc (com.) (2012): Renaissance to Goya. Prints and drawings from Spain. Catálogo de la exposición. Londres. British Museum.

McDonald, Marc (com.) (2013): El trazo español en el British Museum. Dibujos del Renacimiento a Goya. Madrid, Museo Nacional del Prado. Catálogo de la Exposición.

Montaner López, Emilia (1987): Pintura barroca en Salamanca. Salamanca, Universidad Pontificia de Centro de Estudios Salmantinos.

Montaner López, Emilia (1988): «Pintores de la escuela de Madrid en tierras de Guadalajara». En: Wad-alAyara, núm. 15, pp. 387-407.

Muller, Priscilla (com.) (2006): Dibujos españoles en la Hispanic Society of America del siglo de Oro a Goya. Madrid, Museo Nacional del Prado.

Nicolau Castro, Juan (2014): «Un lienzo de la Inmaculada de Matías de Torres en la Colegiata de Talavera de la Reina». En: Archivo Español de Arte, LXXXVII, no 345, pp. 88-92.

Palomino, Acisclo Antonio (1714; ed. de 1947): El museo Pictórico y Escala óptica, ed. Madrid, Aguilar. Pérez Sánchez, Alfonso E. (1965): «Don Matías de Torres». En: Archivo Español de Arte, LVIII, pp. 31-42. Pérez Sánchez, Alfonso E. (1986): Historia del dibujo en España. De la edad media a Goya. Madrid, Cátedra. Pérez Sánchez, Alfonso E (1992): Pintura barroca en España (1600-1750). Madrid. Cátedra.

Pérez Sánchez, Alfonso E. / Navarrete Prieto, B. (1996): «Sobre Herrera el Viejo«. En: Archivo Español de Arte, LXIX, nº 276, pp. 365-387.

Ponz, Antonio (1778): Viage de España. Madrid; vol. V. Citado por ed. (1988), Madrid, Aguilar, vol. 2.

Quesada, José María / Jiménez, América (1996): El arte en Atienza, Guadalajara.

Quesada, José María (1994): «Perspectivas y batallas de Francisco Gutiérrez y Matías de Torres». En: Boletín de la Real Academia de San Fernando, n. 79, pp. 257-288.

Q(uesada), J(osé) M(aría ). (2005): «Inmaculada Concepción». En: Inmaculada. Conferencia episcopal española; catálogo de la exposición. Madrid, pp. 236-258.

Arch. esp. arte, LXXXIX, 353, ENERO-MARZO 2016, 15-28

ISSN: 0004-0428, eISSN: 1988-8511, doi: 10.3989/aearte.2016.02 
VV.AA. (2002): Pintura del Museo Nacional de Escultura. II. Ss. XVII y XVIII, Valladolid.

Zapata, Teresa (2000): La entrada en la Corte de María Luisa de Orleans, Arte y fiesta en Madrid de Carlos II. Madrid, Fundación parea el Estudio del Arte Hispánico.

Zapata Fernández de la Hoz, Teresa (2010): "Unpublised Drawings by Matías de Torres (1635-1711)". En: Master Drawings (Drawings in Spain. In memoriam A. E. Pérez Sánchez), vol. XLVIII, n. 4, pp. 482-497.

Fecha de recepción: 21-VII-2014

Fecha de aceptación: 22-XII-2014

Arch. esp. arte, LXXXIX, 353, ENERO-MARZO 2016, 15-28 ISSN: 0004-0428, eISSN: 1988-8511, doi: 10.3989/aearte.2016.02 